\title{
Inpaichthys kerri n. g. n. sp., um novo peixe caracídeo do alto rio Aripuanã, Mato Grosso, Brasil
}

\author{
J. Géry $\left({ }^{*}\right)$ \\ W. J. Junk (")
}

\section{Resumo}

E descrito um novo peixe caracídeo (Pisces, Cypriniformes, Characoidei) pertencente a um novo gênero Inpaichthys kerri n.g.n.sp., coletado perto da Estação do Núcleo Aripuanã do Instituto Nacional de Pesquisas da Amazônia (INPA), ao Norte de Mato Grosso, Brasil. A espécie nova possui linha lateral incompleta e nadadeira caudal nua. Os dentes são tricuspidados, em duas séries muito irregulares no pré-maxilar; a série interna geralmente composta de somente dois dentes medianos em cada lado. A maxila é dentada em metade de sua borda, geralmente com 7 a 8 dentes tricuspidados e cônicos. Esta dentição, em combinação com outtras características morfológicas descritas no trabalho, difere o Inpaichthys dos outros gêneros da subfamília Tetragonopterinae.

$\mathrm{O}$ pequeno e brilhantemente colorido Tetra, o qual é aqui descrito, foi descoberto perto da Estação do Núcleo Aripuanã, do INPA, ao Norte de Mato Grosso, pela equipe de Ictiologia durante recente expedição de coleta (1). É uma interessante adição para a fauna amazônica, não somente por representar um novo $\mathrm{e}$ raro tipo genérico mas, também, por apresentar um colorido azulado tipo "neon" que poderá torná-lo um interessante peixe de aquário (tal como os "neon-Tetra" Paracheirodon innesi e Cheirodon axelrodi).

\section{Inpaichthys n.g.}

Assim designado em homenagem ao Instituto Nacional de Pesquisas da Amazônia (INPA), Manaus, Brasil.

\section{Espécie Tipo: Inpaichthys kerri n.sp.}

Peixe tetragonopteríneo de pequeno tamanho, caracterizado, como em Hyphessobrycon ("sensu lato"), pela ilnha lateral incompleta e nadadeira caudal nua, mas único entre os Tetragonopterinae por possuir as seguintes características:

Boca subsuperior, dentes tricuspidados em duas séries muito irregulares no pré-maxilar; a série interna geralmente composta de somente dois dentes medianos em cada lado; maxila dentada em metade de sua borda, geralmente com 7 a 8 dentes tricuspidados a cônicos; suborbital grande, inteiro, em contato com o canal preopercular; um único postorbital; nadadeira dorsal claramente atrás do meio do corpo, a qual junto com o pedúnculo relativamente profundo, dá ao peixe o hábito de um Glandulocaudíneo. Contudo, nem glândula caudal nem ganchos são vistos nos peixes que, aparentemente, estão perto de alcançar a maturidade (mais de $25 \mathrm{~mm}$ no comprimento padrão (S.L.)) ou, pelo menos, estão com tamanho médio. Finalmente, a coloração peculiar pode, também, ser um caráier genérico: consiste numa larga e conspícua faixa longitudinal da mandíbula à nadadeira caudal, bastante decurvada em direção ao ventre do peixe (como no Citharinidae africano Neolebias trewavasae, por evolução convergente). In vivo, a metade dorsal do corpo é azulada irıdescente, brilhando como tubos de "neon" (lâmpadas).

(*) - Centre National de la Recherche Scientifique (C.N.R.S.), França, e Instituto Nacional de Pesquisas da Amazônia, Manaus.

(1) - 6 a 18 de novembro de 1976: Grupo composto pelos Drs. Heraldo A. Britski, Naércio A. Menezes, da Universidade de São Paulo, e os autores, além de es iudantes de Pós-Graduação em Ictiologia: Antonio L. de

= Brito. Assad J. Darwich, Barbara A. Robertson, Francisco M. Carvalho, Geraldo M. dos Santos, Ilka M. Paixão, Ivanzir Vieira, Jorge M. Donath, Leoneza H. Soares, Maria G. Soares, Raimunda A. Gonçalves, bem como os pescadores Abel de Souza, Joăo Pena, R. Nona to da Silva, Valdir Costa e os assistentes Pedro Makiyama, Karola Junk e Nildon Ataíde.

Agradecemos à Srta. Bárbara A. Robertson e ao $\mathrm{Sr}$. Ivanzir Vieira pela tradução do manuscrito 


\section{DISCUSSÃo}

Este novo gênero é difícil de situar. De acordo com sua organização geral e a despeito da ausência de glândula caudal, mostra estar próximo a certos gêneros de Glandulocaudinae, como dito acima, e poderia assemelhar principalmente a Coelurichthys tenuis (cf. Géry, 1966), também um Tetra azul o qual, contudo, tem muito mais escamas e diferente dentição. Inpaichthys também recorda Nematobrycon, pequeno Tetra do Nordeste Neotropical (drenagem do Pacífico) pertencente ao grupo Hemibrycon dos Tetragonopteríneos, caracterizado pela presença de um lóbulo caudal mediano apontado (como em certos Phenacogrammus africanos) e ausência de nadadeira adiposa. Os dois gêneros diferem algo consideravelmente em suas dentições.

Outras afinidades poderiam também ser vistas com Hyphes sobrycon melanopleurus Ellis (cf. Géry, 1966), endêmico no rio Tietê, que também poderia ser um novo tipo genérico, e com Rachoviscus crassiceps Myers (de perto do Rio de Janeiro), também raro entre os Tetragonopterinae, situado próximo a Paragoniates et al. Ambas as espécies tem uma dentição diferente de Inpaichthys n.g. . Finalmente, há outro raro e pequeno Tetra que aparenta ter dentição pré-maxilar semelhante a do novo gênero: Bryconel/a pallidifrons Fowler (cf. Géry, 1972), do Alto Amazonas. Inpaichthys difere de Bryconella principalmente no hábito, coloração, maxilar denteado e estrutura dos postorbitais, e não parece pertencer à mesma linha filogenética.

\section{Inpaichthys kerri n.sp.}

Assim designado em homenagem ao Dr. Warwick Estevam Kerr. Diretor do INPA.

\section{HOLÓTIPO:}

Macho (?) $28 \mathrm{~mm}$ comprimento padrão (S.L.) coletado em 14 de novembro de 1976 num pequeño igarapé pertencente ao sistema do igarapé Queimada (atualmente igarapé do Aeroporto), cerca de duas horas da Cidade de
Humboldt (Núcleo Aripuanã), na futura estrada para Juruena, alto rio Aripuanã. INPA $-\mathrm{N} .^{\circ}$ 7600080 .

PARÁTIPOS:

110 exemplares, o maior com comprimento padrão de $26 \mathrm{~mm}$, coletado com o tipo (5 exemplares corados com alizarina);

2 exemplares, $19-23 \mathrm{~mm}$ de comprimento padrão, igarapé do Aeroporto, ca. $3 \mathrm{~km}$ da Cidade de Humboldt, mesma drenagem, coletados a 9 de novembro de 1976;

2 exemplares, $24-25 \mathrm{~mm}$ de comprimento padrão, igarapé da Chapada (afluente do igarapé Aeroporto), mesma drenagem, coletados em 11 de novembro de 1976;

4 exemplares, o maior com $17 \mathrm{~mm}$ de comprimento padrão, Igarapé Taboca, tributário alto rio Aripuanã, ca. $50 \mathrm{~km}$ acima de Aripuanã (Cidade Humboldt), coletados em 12 de novembro de 1976;

1 exemplar, comprimento padrão de 19,5 $\mathrm{mm}$, Igarapé Jenipapo, tributário do alto rio Aripuanả, cerca de $70 \mathrm{~km}$ acima da Cidade de Humboldt, coletado em 14 de novembro de 1976.

NOTA SOBRE O "LOCUS TYPICUS":

A estação do INPA foi implantada na Cidade de Humboldt, Aripuanã, MT, acima das grandes cachoeiras de Dardanelos e Andorinhas, no alto rio Aripuanã, tributário do rio Madeira. Todas as coletas foram feitas em pequenos igarapés acima das cachoeiras. Coordenadas $10^{\circ} 10^{\prime} \mathrm{S}, 59^{\circ} 25^{\prime} \mathrm{W}$.

\section{DESCRIÇÃO:}

(Veja Tabela 1 para detalhes das proporçốes dos 25 maiores exemplares).

Corpo moderadamente alongado, os perfís dorsal e ventral iguais, altura máxima 3,0-3,5 no comprimento padrão (média 3,28 , coeficiente de variação $\left.v=\frac{100 \mathrm{~s}}{\bar{x}}=4,6\right)$; altura do pedúnculo variável, $0,93-1,36$ no comprimento, $\bar{x}=1,12, v=10,6$, nadadeira dorsal 
claramente posierior ao meio do corpo, distância pré-dorsal $0,86-0,97$ na distância pós dorsal ( $\bar{x}=0,92, v=3,15$ ), fórmula ii9, às vezes com um raio rudimentar não ramificado na frente, portanto (i) ii9, o qual não é comum nos Characidae; peitorais baixas, mais curtas que o comprimento da cabeça, geralmente não alcançando a nadadeira seguinte; nadadeira anal começando sob os primeiros raios da dersal ou, algumas vezes, à frente deles, fórmula (i) iii, 22-26 (último raio ramificado dividido na base) .

70 exemplares escolhidos ao acaso apresentaram a seguinte distribuição de raios ramificados: 22, 10 exemplares; 23, 34 exemplares; 24, 17 exemplares; 25, 7 exemplares: 26, 2 exemplares; uma única série de escamas no primeiro terço da base da anal; sem lobos visíveis; borda da anal arredondada; não foram observados ganchos sexuais; nadadeira caudal com lobos iguais, bem furcada, sem lobo mediano: fórmula como usual em Characoidei; i $9 / 8$ i; vii ou viii raios acessórios acima e v-vi abaixo; sem raios caudais separados do restante da nadadeira; sem glândulas ou ganchos separados (mais de 70 exemplares examinados); sem escamas nos lobos exçto uma grande em cada lobo, caudal; nadadeira adiposa presente, estreita, de tamanho moderado; sem espinhos interhemais, sem pseudotímpano ou "hiatus" humeral, região preventra! arredondada mas a pós ventral claramente na forma de quilha; pedúnculo sem quilha.

Escamas do tipo Hemigrammus, delgadas, difíceis para observar; linha lateral sempre incompleta, com tubos nas primeiras 6 ou 7 escamas; geralmente $34-36$ escamas em série Ionjitudinal, 11 ou 12 em séries transversais entre as nadadeiras dorsal e ventral (geralmente $6 / 1 / 4 ; 13$ ou 14 em série prédorsal, regular, ca. $16 \mathrm{em}$ torno ao pedúnculo caudal.

Cabeça (Fig. 1) relativamente curta, comprimento (sem membrana) 3,75-4,1 no comprimento padiăo $(\bar{x}=3,95, V=2,4)$, perfil dorsal um pouco convexo; interorbital 2,6-3,2 no comprimento da cabeça $(\bar{x}=2,88$, $v=5,3)$; olho grande, redondo, sem pálpebra adiposa, diâmetro vertical $2,55-3,15$ no comprimento da cabeça $(\bar{x}=2,8, v=5,9)$; maxilar bastante obliquo (devido à posição relativamente superior da boca) alcançando a

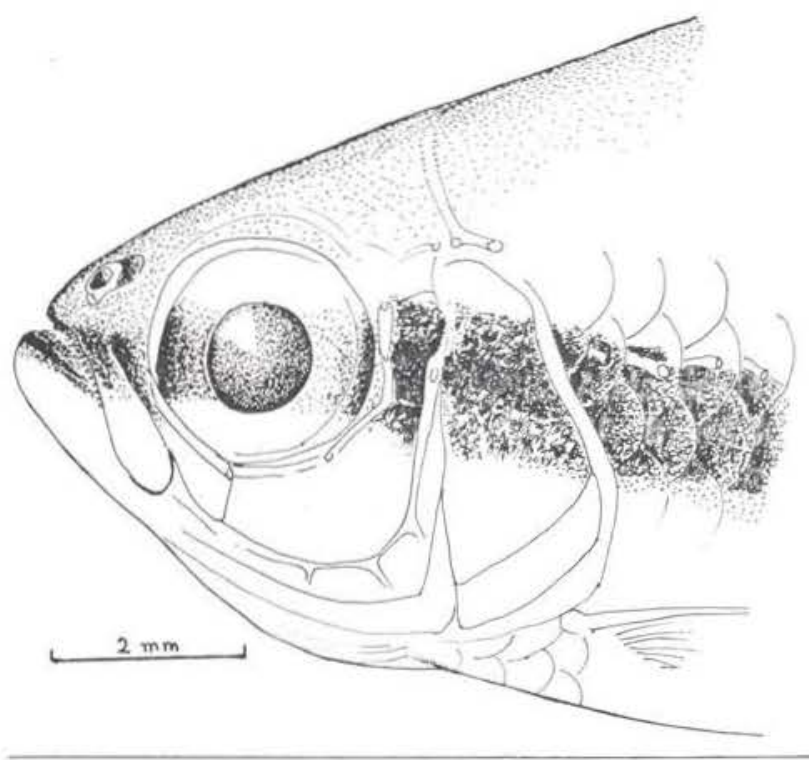

Fig. 1 - Cabeça de Inpaichthys kerri n.g.n.sp.

margem anterior do olho, geralmente não alcançando o nivel da pupila, comprimento aparente $3,2-4,05$ no comprimento da cabeça $(\bar{x}=3,66, v-7,1)$, focinho curto e arredondado, metade do diâmetro do olho em projeção ou menos, 4-6 no comprimento da cabeça $(\bar{x}=4,73, v=8,7)$; mandibula proeminente, boca formando ângulo com mais que $45^{\circ} \mathrm{com}$ o eixo do peixe; narinas próximas; fontanelas presentes, anterior geralmente alcançando o nivel do meio do olho; primeiro suborbital reduzido; segundo e terceiro suborbitais bem desenvolvidos, o terceiro (grande suborbital) em contato com o canal do preopérculo exceto em sua parte superior; quarto circumorbital (primeiro postorbital) aito e estreito não recobrindo totalmente a face; sem outro postorbital, sem supraorbital. Rastros branquiais normais, 6 a 7 acima e 10 ou 11 abaixo no primeiro arco branquial.

Mandibula (Fig. 2) do tipo tetragonopteríneo, isto é, com um bem desenvolvido pro. cesso prémaxilar ascendente e uma maxila bem desenvolvida; os dentes maiores principalmente tricuspidados tornando-se cònicos nos lados da mandíbula como também na parte distal da maxila.

Os dentes prémaxilares estão em duas fileiras na maior parte dos espécimes examinados, isto é, com uma fileira externa de, geralmente, 5 dentes tricuspidados na parte laterai 


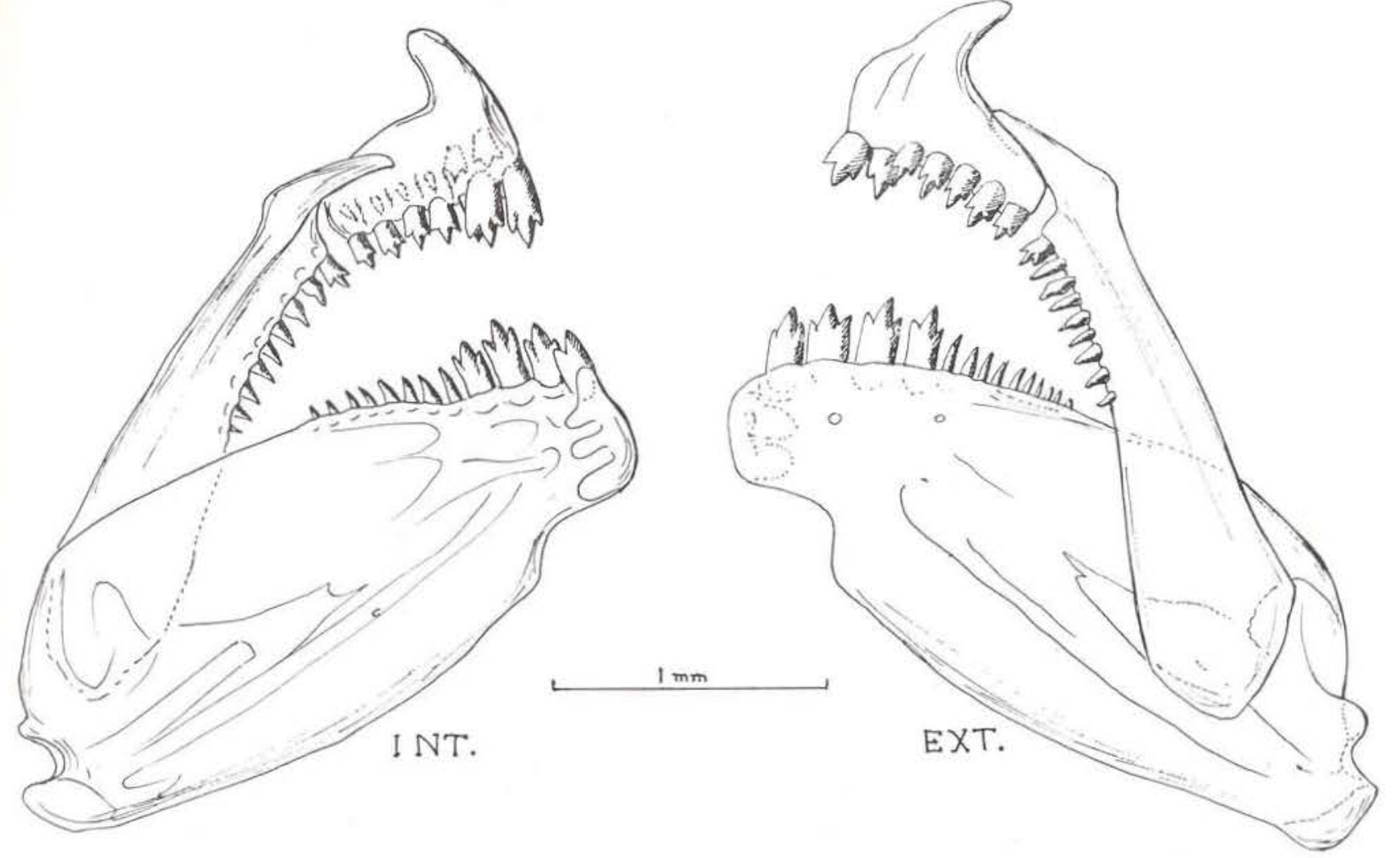

Fig. 2 - Maxilares de Inpaichthys kerri n.g.n.sp., vista lateral esquerda interna e externa.

do osso (deixando um "buraco" em seu quinto mesial), e uma fileira interna de dois dentes um pouco maiores na parte mesial do osso; em alguns dos espécimes há 6 dentes externos e somente um interno ou, algumas vezes, 4 externos e 3 dentes internos; num caso houve aparentemente 8 dentes numa fileira única e mais ou menos regular; no geral, a distribuição dos dentes prémaxilares parece seguir as mesmas regras de Bryconella, já mencionadas na discussão do gênero; maxilar mais que duas vezes o comprimento do prémaxilar, com uma apófise bem desenvolvida e 7 ou 8 dentes (raramente 6 ou 9) ao longo da metade de sua borda, na maior parte cônicos, poucos tricuspidados perto do ângulo; mandíbula aparentemente sem caracteres incomuns, com quatro dentes tricuspidados em sua parte anterior, seguindo-se a estes até 10 dentes menores, cônicos.

Em vida, quando excitados e iluminados pe'o sol, a coloração do peixe é impressionante: a faixa lateral é azul escura e o peixe todo é banhado por uma cor azul-violeta que se estende até a nadadeira adiposa; há uma mancha azul brilhante acima do opérculo; a extremidade da nadadeira dorsal é avermelhada e a nadadeira caudal algo amarelada. (Fig. 3) .

Imediatamente, após a preservação em formalina, a cor azul desaparece; os flancos tornam-se um vivo alaranjado (fenômeno similar é visto em Hemigrammus coeruleus Durbin, sendo que é muito menos azul em vida e muito mais vermelho em formalina), e a faixa lateral que se estende até a nadadeira caudal fica preta. Um pouco da coloração preta também aparece nas extremidades das nadadeiras pares, bem como na margem da anal. (Fig. 5).

\section{DADOS ECOLÓGICOS:}

Inpaichthys kerri vive nas áreas ensolaradas e de correnteza dos igarapés (Fig. 4) pertencentes ao sistema fluvial do Queimada, tributário do alto rio Aripuanã, acima das cachoeiras. São águas pobres em eletrólitos, um pouco 


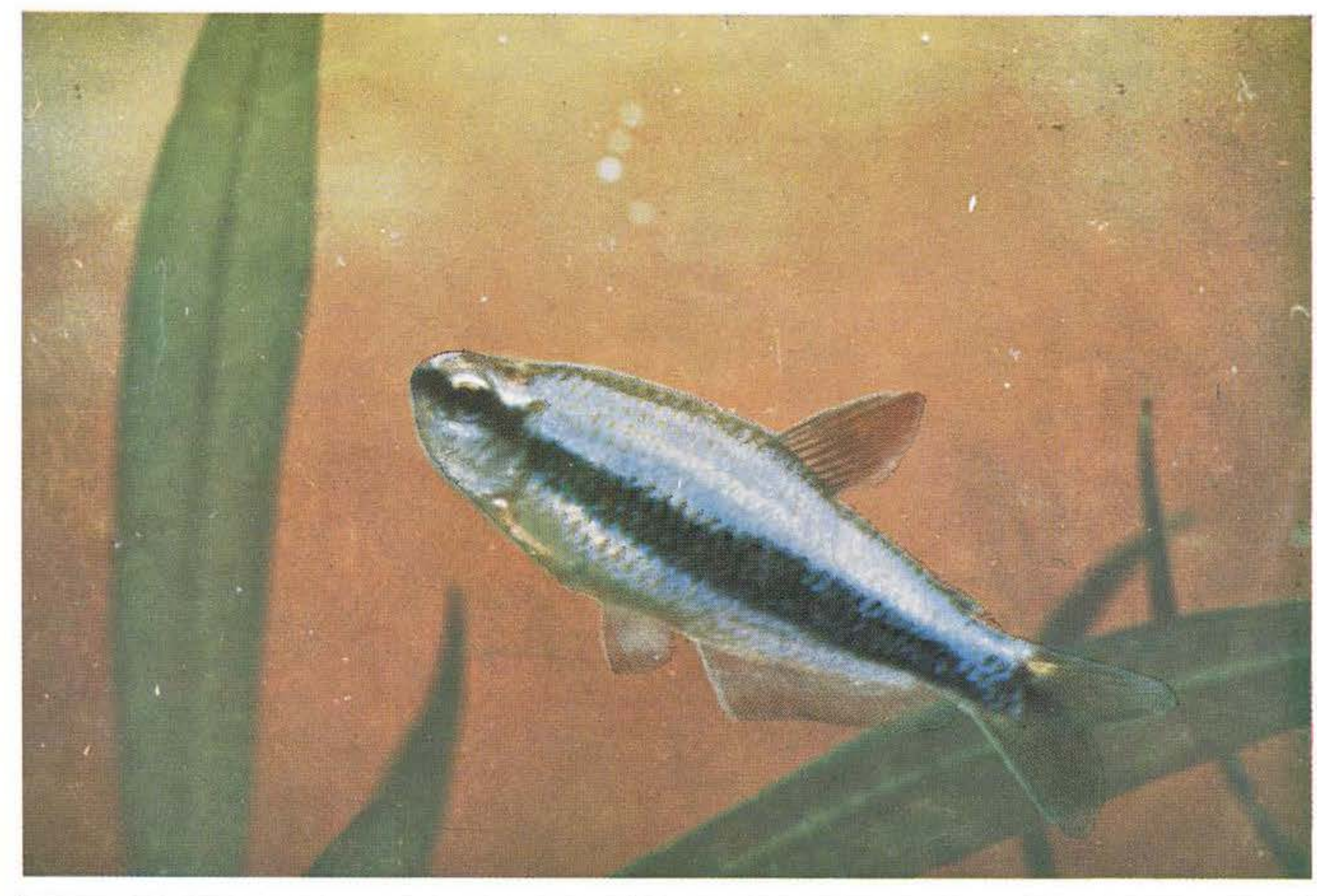

Fig. 3 - Inpaichthys kerri n.g.n.sp. in vitro.

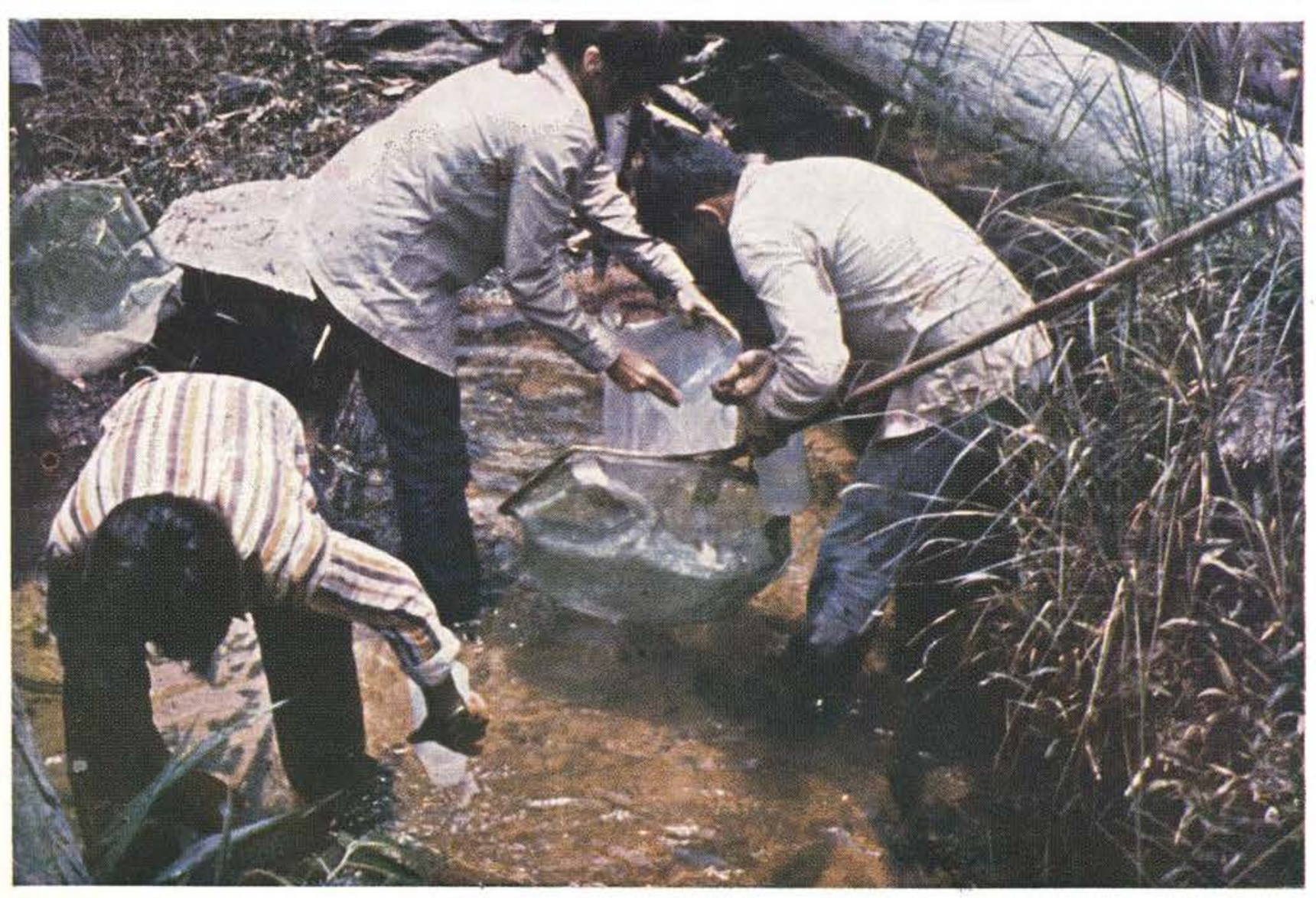

Fig. 4 - Local típico de Inpaichthys kerri n.g.n.sp. 


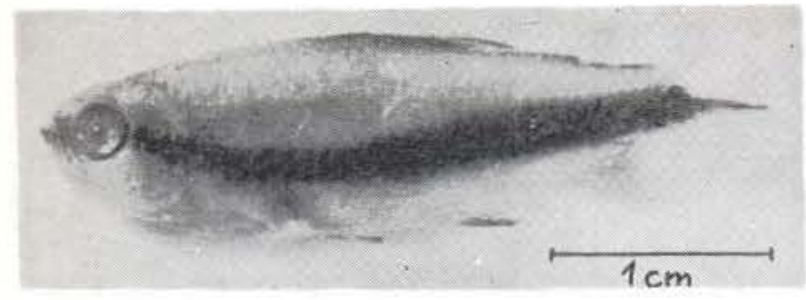

Fig. 5 - Inpaichthys kerri n.g.n.sp., holótipo.

ácidas. Durante o período ae coleta os valores de condutividade elétrica variaram entre 9 a $11 \mu \mathrm{S} 20$, valores de $\mathrm{pH}$ entre 6,0 a 6,8 nas diferentes estações de coleta. Estes igarapés de floresta são habitados por uma rica e abundante fauna, com os quatro principais grupos amazônicos (Caracoídeos, Gimnotoídeos, Siluroideos e Ciclideos) bem representados. $\mathrm{Pa}$ recem viver em associação com outro pequeno Tetra que apresenta uma faixa lateral reta, $\left({ }^{2}\right)$ Hyphessobrycon cf. cachimbensis Travassos, e geralmente é menos abundante que o último, na proporção $1 / 200$ ou menos, embora possa ser bastante abundante em alguns pontos. Conforme seu comportamento em aquário, Inpaichthys kerri, é peixe de cardume, alimentando-se principalmente na superfície de insetos caidos; extremamente ativo, não é tímido nem sensivel ao transporte ou mudanças de água. C. reprodução năo foi ainda observada. A exibição dos machos parece ocorrer próximo ao fundo.

\section{SUMMARY}

A new little Tetra (Pisces, Cypriniformes, Characoidei) belonging to a new genus Inpaichthys kerri n.g.n.sp., is described. It was collected near the field station of Instituto Nacional de Pesquisas da Amazônia (INPA), Nucleo Aripuană, in the north of Mato Grosso-Brazil. This species has the lateral line incomplete and the caudal fin naked. The teeth are tricuspidate in two very irregular rows on the premaxilla. The inner row is usually composed of only 2 median teeth on each side. The maxilla is toothed on half of its edge, with usually 7 or 8 tricuspidate to conical teeth. This dentition in combination with other morphological characters described in the paper differentiate Inpaichthys from the other genera of the subfamily Tetragonopterinae.

\section{BIBLIOGRAFIA CITADA}

GÉRY, J

1966 - A review of certain Tetragonopterinae (Characoidei), with the description of two new genera. - Ichthyologica The Aquarium Journal, 37(5):211-236.

1972 - Corrected and supplemented description of certain characoid fishes described by Henry W. Fowler, with revisions of several of their genera. Stud. Neotrop. Fauna, 7:1-35.

MYERS, G.S

1926 - Eine neue Characinidengattung der Unterfamilie Cheirodontinae aus Rio de Janeiro, Brasilien. - Blätter Aquar. Terrarienk., 37(24):566-567 (Rachoviscus crassiceps).

(2) - Vale mencionar que no Gabão (África), uma associação semelhante existe entre Neolebias trewavasae, a qual tem uma faixa lateral decurvada e Neolebias unitaeniatus ssp. a qual tem uma faixa lateral reta, como se fatores similares evolutivos estivessem agindo em ambos os lados do oceano. 


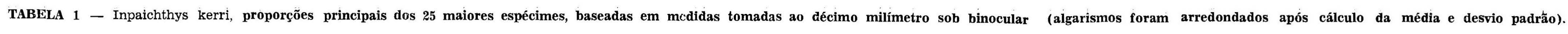

\begin{tabular}{|c|c|c|c|c|c|c|c|c|c|c|c|c|c|c|c|c|c|c|c|c|c|c|c|c|c|}
\hline N.o & $\begin{array}{c}1 \\
\text { Holó. } \\
\text { tipo }\end{array}$ & 2 & 3 & 4 & 5 & 6 & 7 & 8 & 9 & 10 & 11 & 12 & 13 & 14 & 15 & 16 & 17 & 18 & 19 & 20 & 21 & 22 & 23 & 24 & $\begin{array}{l}\text { FAIXA, MÉDIA E } \\
\text { DESVIO PADRAO }\end{array}$ \\
\hline $\begin{array}{l}\text { Comprimento padrão (S.L.) } \\
\text { em mm }\end{array}$ & 28 & 26,5 & 26 & 26 & 26 & 25 & 25 & 25 & 25 & 24,5 & 24,5 & 24,5 & 24 & 24 & 24 & 23,5 & 23,5 & 23,5 & 23 & 23 & 23 & 22,5 & 22,5 & 22,5 & $22,5-28$ \\
\hline SL/altura do corpo & 3,35 & 3,05 & 3,25 & 3,1 & 3,05 & 3,0 & 3,1 & 3,35 & 3,45 & 3,2 & 3,2 & 3,4 & 3,2 & 3,2 & 3,45 & 3,2 & 3,35 & 3,5 & 3,25 & 3,45 & 3,45 & 3,35 & 3,5 & 3,15 & $3,4 \begin{array}{c}3,0-3,5 \\
\bar{x}=3,28 ; \quad s=0,15\end{array}$ \\
\hline Postdorsal/Prédorsal & 0,92 & 0,92 & 0,93 & 0,9 & 0,96 & 0,97 & 0,9 & 0,96 & 0,88 & 0,3 & 0,86 & 0,89 & 0,94 & 0,94 & 0,94 & 0,94 & 0,93 & 0,94 & 0,95 & 0,96 & 0,94 & 0,93 & 0,92 & 0,89 & $0,89 \begin{array}{r}0,86-0,97 \\
\bar{x}=0,92 ; \quad s=0,03\end{array}$ \\
\hline $\begin{array}{l}\text { Comprimento pedúnculo/ } \\
\text { altura ped. }\end{array}$ & 1,03 & 1,13 & 1,2 & 0,93 & 1,13 & 1,0 & 1,06 & 1,04 & 1,22 & 0,93 & 1,36 & 0,93 & 1,15 & 1,18 & 1,03 & 1.19 & 1,3 & 1,28 & 1,28 & 1,2 & 1,12 & 1,08 & 1,04 & 1,08 & $1,04 \bar{x} \begin{array}{r}0,93-1,36 \\
\bar{x}=1,12 ; \mathrm{s}=0,12\end{array}$ \\
\hline $\mathrm{SL} /$ Comprimento cabeça & $\measuredangle, 0$ & 4,1 & 3,95 & 3,9 & 4,0 & 3,8 & 3,9 & 4,1 & 4,1 & 3,85 & 4,0 & 3,95 & 4,0 & 4,0 & 3,75 & 3,85 & 4,0 & 4,0 & 3,95 & 3,85 & 4,05 & 3,2 & 3,95 & 4,0 & $3,95 \begin{array}{r}3,75-4,1 \\
\bar{x}=3,95 ; \quad s=0,1\end{array}$ \\
\hline Cabeça/Interorbital & $\ddot{2,8}$ & 2,85 & 2,75 & 3,05 & 2,6 & 2,85 & 2,9 & 2,9 & 3,2 & 3,05 & 3,2 & 2,8 & 2,75 & 2,85 & 3,05 & 2,75 & 2,95 & 2,95 & 2,75 & 2,85 & 2,6 & 2,95 & 3,0 & 2,8 & ${ }^{2,85} \begin{array}{c}2,6-3,2 \\
\bar{x}=2,88 ; \quad s=0,15\end{array}$ \\
\hline Cabeça/Olha & 3,05 & 3,1 & 2,85 & 3,0 & 2,85 & 3,15 & 2,7 & 2,65 & 2,65 & 3,05 & 2,65 & 2,7 & 2,75 & 2,75 & 2,9 & 2,9 & 2,8 & 2,6 & 2,75 & 2,85 & 2,6 & 2,7 & 2,6 & 2,55 & $2,7 \begin{array}{c}2,55-3,15 \\
\bar{x}=2,8 ; \quad s=0,16\end{array}$ \\
\hline Cabeça/Maxila & 3,9 & 3,8 & 3,9 & 2,95 & 3.6 & 2,65 & 3,55 & 3,2 & 3,2 & 3,75 & 3.6 & 3,65 & 3,35 & 3,55 & 4,0 & 3,6 & 3,3 & 3,45 & 3,6 & 3,75 & 4,05 & 3,95 & 4.05 & 3,75 & $3,35 \begin{array}{c}2,65-4,05 \\
\bar{x}=3,66 ; s=0,26\end{array}$ \\
\hline Cabeça/Focinho & 5,0 & 5,0 & 4,4 & 5,15 & 5,0 & 6,0 & 4,9 & 4,7 & 4,7 & 4,9 & 4,7 & 4,75 & 4,0 & 4,3 & 4,25 & 5,1 & 4,9 & 4,55 & 4,45 & 4,6 & 4,75 & 4,9 & 4,4 & 4,0 & $4,75 \begin{array}{c}4,0-6,0 \\
\bar{x}=4,73 ; s=0,41\end{array}$ \\
\hline
\end{tabular}

